NISTIR 7908

\title{
A Sensor Ontology Literature Review
}

Roger Eastman Craig Schlenoff

Stephen Balakirsky

Tsai Hong

http://dx.doi.org/10.6028/NIST.IR.7908

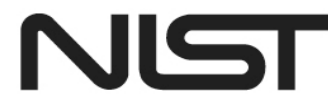
National Institute of Standards and Technology U.S. Department of Commerce 
NISTIR 7908

\title{
A Sensor Ontology Literature Review
}

\author{
Roger Eastman \\ Craig Schlenoff \\ Stephen Balakirsky \\ Tsai Hong \\ Intelligent Systems Division \\ Engineering Laboratory
}

http://dx.doi.org/10.6028/NIST.IR.7908

April 2013

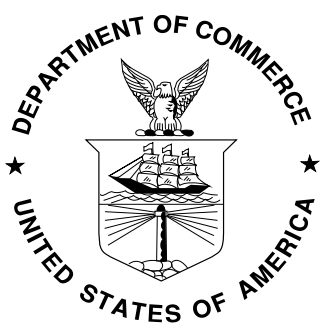

U.S. Department of Commerce Rebecca Blank, Acting Secretary

National Institute of Standards and Technology Patrick D. Gallagher, Under Secretary of Commerce for Standards and Technology and Director 


\title{
A Sensor Ontology Literature Review
}

\author{
Roger Eastman, Craig Schlenoff, Stephen Balakirsky, Tsai Hong
}

\begin{abstract}
The purpose of this paper is to review existing sensor and sensor network ontologies to understand whether they can be reused as a basis for a manufacturing perception sensor ontology, or if the existing ontologies hold lessons for the development of a new ontology. We develop an initial set of requirements that should apply to a manufacturing perception sensor ontology. These initial requirements are used in reviewing selected existing sensor ontologies. This paper describes the steps for 1) extending and refining the requirements; 2) proposing hierarchical structures for verifying the purposes of the ontology; and 3) choosing appropriate tools and languages to support such an ontology. Some languages could include OWL (Web Ontology Language) [1] and SensorML (Sensor Markup Language) [2]. This work will be proposed as a standard within the IEEE Robotics and Automation Society (RAS) Ontologies for Robotics Automation (ORA) Working Group [3].
\end{abstract}

\section{Overview of Sensor Ontology Effort}

Next generation robotic systems for manufacturing must perform highly complex tasks in dynamic environments. To improve return on investment, manufacturing robots and automation must become more flexible and adaptable, and less dependent on blind, repetitive motions in a structured, fixed environment. To become more adaptable, robots need both precise sensing for parts and assemblies, so they can focus on specific tasks in which they must interact with and manipulate objects; and situational awareness, so they can robustly sense their entire environment for long-term planning and short-term safety.

Meeting these requirements will need advances in sensing and perception systems that can identify and locate objects, can detect people and obstacles, and, in general, can perceive as many elements of the manufacturing environment as needed for operation. To robustly and accurately perceive many elements of the environment will require a wide range of collaborating smart sensors such as cameras, laser scanners, stereo cameras, and others. In many cases these sensors will need to be integrated into a distributed sensor network that offers extensive coverage of a manufacturing facility by sensors of complementary capabilities.

To support the development of these sensors and networks, the National Institute of Standards and Technology (NIST) manufacturing perception sensor ontology effort looks to create an ontology of sensors, sensor networks, sensor capabilities, environmental objects, and environmental conditions so as to better define and anticipate the wide range of perception systems needed. The ontology will include: 
- detailed standard knowledge representations of sensor physical dimensions, weight, resolution, associated system performance, and operating conditions;

- representations of system capabilities, to categorize the functions that individual and groups of sensors can perform; and

- representations of sensor embeddings in sensor networks and the manufacturing environment.

The ontology will have three primary objectives. The first is to form the basis for new measurement methods to evaluate each perception system's (sensors and algorithms) ability to perform their required tasks. Prototypes of these perception systems are being developed but science-based approaches to accurately and traceably measure their performance do not exist. The NIST sensor ontology effort is part of a larger project to develop the metrics and methods that underpin such approaches. Its focus will be the ability to detect people and objects as they move about the workspace. The project will build a testbed in which to conduct experiments to assess that ability. Project results will provide scientific foundations for new standards that enable the use of perception systems in manufacturing applications. One aspect of the testbed will be to test the systems ability to locate people. For this, the project will work to create ground truth for tracking the location of people, and metrics for evaluating systems against this ground truth. The sensor ontology will help to categorize sensors and their capabilities.

The second objective will be to support the design and calibration of networks by reasoning about required capabilities. By mapping sensors into capabilities and measured performance, the ontology will enable a facility designer to consider required capabilities at an abstract level and reason about the best performing and lowest cost combination of sensors to meet facility needs.

Similarly, the third objective is to enable an operating robotic system to reason about the available and running sensor systems to apply them to current perception needs, querying and retasking sensors as needed.

The purpose of this document is to review existing sensor and sensor network ontologies to understand whether they can be reused as a basis for a manufacturing perception sensor ontology, or if the existing ontologies hold lessons for the development of a new ontology. Before reviewing the existing ontologies for their application to manufacturing, this document states requirements that should apply to a manufacturing perception sensor ontology. Section 2 develops these requirements, and Section 3 uses these requirements in reviewing selected existing sensor ontologies. Subsequent documents will focus on a detailed set of requirements for the manufacturing sensor ontology and the contents/structure of the manufacturing sensor ontology once it is developed. 


\section{High Level Requirements for a Manufacturing Sensor Ontology}

While sensors have similar characteristics across applications, from earth science to military to manufacturing, a sensor ontology for manufacturing will have unique needs and uses. Sensors will be (typically) installed in an indoor facility, used to perceive objects and manufacturing processes, designed to meet appropriate power and operating conditions, and repurposed as product lines undergo change. Key here are the required capabilities of a sensor to perceive objects and processes as distinct from other applications. In a Geographic Information System (GIS) earth science application, sensors usually measure basic physical properties (temperature, air pressure) meant to represent large areas, while in manufacturing a sensor may need to perceive the position and identity relatively small parts. It may be possible to integrate sensor ontologies from different domains, but the integrated representation should be suitable in each.

The intended purposes of the manufacturing perception sensor ontology are to:

- Provide a basis for measurement techniques to evaluate system performance.

- Support design of sensor networks for manufacturing applications.

- Categorize and organize calibration techniques for sensors and sensor networks.

- Reason about available sensors and capabilities to meet goals during operation.

- Create mechanisms and languages for querying sensors.

- Provide logs and data provenance for potential legal and regulatory review.

Based on these purposes, we can consider the sensor and system attributes that should be recorded in the ontology. Table 1 gives a potential list of attributes that could be associated with sensors and sensor systems. These are a high-level set of requirements and will be refined in subsequent phases of the effort.

The output of the sensors and sensor systems are important, and are described by five attributes in the table: Immediate data, Derived data, Algorithms, Integration/fusion and Capabilities. Immediate data describes the raw, unfiltered data; Derived data describes data and interpretations computed from the original data; Algorithms describes the different, possibly alternative, procedures for computing derived data; Integration/fusion describes derived data that comes from multiple sensor sources; and Capabilities describes the functional applications to which immediate and derived data can be put. It may be possible to collapse the five levels into fewer, but the current five are a working set of categories. It may be possible to put data into a classic hierarchical model, such raw data points, derived geometric entities like planes and surfaces, and semantic interpretations like objects and behaviors. 
Table 1 - General Attributes for Sensors and Sensor Systems/Networks

\begin{tabular}{|l|l|}
\hline Attributes & Comments \\
\hline Physical & Power, weight, size \\
\hline Operating conditions & Environmental conditions required for operation \\
\hline Immediate data & $\begin{array}{l}\text { Characteristics of data, resolution (time/spatial/latency/frame } \\
\text { rate/other) }\end{array}$ \\
\hline Derived data & Results computed from raw data, both physical and semantic \\
\hline Algorithms & Alternative algorithms for producing derived data \\
\hline Integration/fusion & Data produced by combining data from multiple sensors \\
\hline Capabilities & Functional applications of raw and derived data \\
\hline Communication & Physical and logical protocols, and interoperability \\
\hline Processing & On board processing power of sensors and network nodes \\
\hline Calibration & Individual and joint sensor calibration information/algorithms \\
\hline Provenance & Record of sensor and processing history of raw and derived data \\
\hline Confidence & Levels of confidence in derived data \\
\hline
\end{tabular}

Algorithms are presented as a distinct attribute since a particular interpretation of the data, such as the presence of a person, may be computed in different ways from the raw data. For purposes of tracking provenance and confidence, it may be important to understand the processing steps that lead to the conclusion.

Capabilities are the interpretation, or affordances, of data as related to the goals of the overall robotic systems. The presence of a planar surface may be the derived data computed from a depth sensor; the fact that the surface affords a robot a platform for wheeled motion is a capability the sensor system provides the robot.

As a first step to combining these attributes into a hierarchy, Table 2 presents a basic outline.

In addition to the concepts and attributes that must be supported by the ontology, a second question to answer is the nature of the ontology itself. What are the syntactic and semantic requirements for the ontology representation?

Given that sensor technologies are constantly changing, and that a manufacturing ontology should be applicable to different industrial domains, the ontology should be readably extensible. In particular, the capabilities that must be described will need to be adapted to the application domain. Based on the previously stated purposes for the manufacturing ontology, we know it should support reasoning with suitably complete and consistent descriptive logic. To describe dynamic environments and actions, the logic should manage temporal elements. 
Table 2 - Sensor Requirements

\begin{tabular}{|c|c|}
\hline \multicolumn{2}{|r|}{ Proposed } \\
\hline \multirow{3}{*}{$\begin{array}{ll}\text { 1. } & \text { Three } \\
\text { Perception } \\
\text { Levels }\end{array}$} & 1.1 Level 1: Raw data level \\
\hline & 1.2 Level 2: Primitive level \\
\hline & 1.3 Level 3: Object level \\
\hline \multirow{7}{*}{$\begin{array}{ll}\text { 2. } & \text { Sensor } \\
& \text { Characteristics }\end{array}$} & 2.1 Operating conditions \\
\hline & 2.2 Resolution \\
\hline & 2.3 Power, Weight, Size \\
\hline & 2.4 Sensor hierarchy \\
\hline & 2.5 Sensor communication \\
\hline & 2.6 Raw data confidence \\
\hline & 2.7 Onboard processing \\
\hline \multirow{7}{*}{$\begin{array}{l}\text { 3. Sensor } \\
\text { Network }\end{array}$} & 3.1 Sensors \\
\hline & 3.2 Algorithms \\
\hline & 3.3 Capabilities \\
\hline & 3.4 Communications/Interoperability \\
\hline & 3.5 Calibration algorithms \\
\hline & 3.6 Network topology/geometry \\
\hline & 3.7 Processing resources \\
\hline \multirow{6}{*}{ 4. Algorithms } & 4.1 Provenance \\
\hline & 4.2 Derived data confidence \\
\hline & 4.3 Input requirements \\
\hline & 4.4 Capabilities \\
\hline & 4.5 Performance \\
\hline & 4.6 Hardware/OS requirements \\
\hline
\end{tabular}

\section{Review of Sensor Ontologies}

In reviewing the literature on sensor ontologies, we selected seven research programs of interest and one review article. In this section we will look at each program and its relevance to the NIST sensor ontology project.

\subsection{Compton et al. Sensor Ontology Survey}

The survey article by Compton [4] gives a useful start at terminology appropriate across sensor ontologies, and assisted in constructing this review. It surveys 12 ontologies published from 2001 to 2009, looking at the range and expressive power of the ontologies as well as concepts these ontologies cannot describe accurately. The survey looks in particular at sensor networks and the use of ontologies in their 
operation and description. Many existing sensor ontology articles, and the Compton et al survey, are focused on the Semantic Web and the efforts to support autonomous agents that can find and use sensors attached to the Internet. This is a much larger and amorphous environment than a fixed manufacturing facility network, but similar concepts are needed for both.

Some of the terminology defined in the survey includes:

Sensor: a source that produces a value representing a quality of a phenomenon. It includes a range of instruments, including transducers, sensor devices, and computations. Comment: in this case, sensors are defined as combinations of transducers and processing. In some cases, sensors are defined more restrictively as transducers.

Sensor network: networks of connected sensors and associated devices. Nodes (devices with attached sensors) interact to form a sensor network, and deliver data, through a gateway, to a base station. The base state and associated services then offer live and historical data, analysis, interpretation, and prediction.

Semantics: The semantic approach to information systems design uses declarative descriptions of information and processing units, allowing (semi-)automatic satisfaction of declaratively described requirements. Declarative descriptions enable both domain-independent and domain-specific reasoning of various forms (logic-based or otherwise) to be applied in processes such as entity identification, search, and query and workflow generation. Semantics enables reasoning, including search, logical reasoning, and domain reasoning. Semantics serve a dual role: it is part mark-up and elicitation of information and part logic for reasoning.

Semantic Sensor Network: requires declarative specifications of sensing devices, the network, services, and the domain and its relation to the observations and measurements of the sensors and services. Processing tools, logical and otherwise, can then be used to answer queries, infer further information, search for and identify particular resources, or generate workflows, all of which might require reasoning and inference in analyzing the specifications, links between entities and data, allowing users to develop, use, and adapt sensor networks, while abstracting away the low-level details and difficulties of the network and its multiple devices.

Semantic Sensor Network Architecture: multiple layers of semantics and technology to provide infrastructure and services.

Three Layer Architecture: a particular model of data, processing, and application, to support network-internal processing, inference and integration, and services respectively.

Moving from the survey article to specific ontologies, below are seven research programs with a short review of each. 


\subsection{Ontology-Driven Adaptive Sensor Networks}

Avancha et al. [5] describe an ontology for adaptive sensor networks, with nodes that react to available power and environmental factors, calibrate for accuracy, and determine suitable operating states. The focus of this ontology is on adapting a wireless sensor network to current operating conditions while maintaining calibration and communication. The usefulness of this work for the NIST effort is in the concepts related to a sensor network and communication.

\subsection{A Formal Pedigree Ontology for Level-One Sensor Fusion}

Matheus et al. [6] is intended for maintaining provenance of data from multiple sensors in a naval military application, so a user can understand how to judge and interpret sensor results. By associating the originating sensor output with data already represented in the ontology, the ontology can let users decide which of multiple sensor streams to trust. For example, if multiple sensors are providing tracks of a ship or airplane, the ontology can describe appropriate metadata so the system can annotate track displays with provenance and confidence information. The usefulness of this work for the NIST effort is in the provenance concepts.

\subsection{An Ontology-Centric Approach to Sensor- Mission Assignment}

Gomez et al [7] and Preece et al [8] describe an ontology for automatically selecting sensors for task assignment based on sensor fitness for the task description. The system was designed to fulfill military missions given high-level descriptions of needed capabilities and available resources. These papers also describe reasoning algorithms to carry out the matching of sensor capabilities and transportation platforms to the mission under resource constraints. The emphasis is on premission planning, not during mission execution. The usefulness of this work is in the mission to sensor matching for system design.

\subsection{A Universal Ontology for Sensor Networks Data}

Eid et al $[9,10]$ propose a two-tier framework for a sensor ontology. In their framework, the sensor hierarchy, data, and extension ontologies (lower tier) all reference the Standard Upper Merged Ontology (SUMO) (upper tier). SUMO [11] is the Suggested Upper Merged Ontology, which provides common high-level concepts that can be extended to produce domain specific ontologies, such as a sensor ontology. The lower tier ontology is divided into three parts: a Sensor Hierarchy Ontology (SHO) to describe transducer classes and attributes; a Sensor Data Ontology (SDO) to describe data; and a format for Extension Plug-in Ontologies 
(EPO) that allows developers to integrate domain-specific elements with the universal ontology. The usefulness of this article for the NIST effort is to provide a framework for integrating and extending ontologies, and using SUMO in a sensor ontology.

\subsection{The Semantic Sensor Network Ontology}

Neuhaus and Compton [12] is a generic ontology for describing sensors and deployments to be used in data integration, search, classification, and workflows. The ontology comprises four core clusters of concepts: (1) those concepts describing the domain of sensing (Feature); (2) those describing the sensor (Sensor); (3) those describing the physical components and location of the sensor (SensorGrounding); and (4) those describing functions and processing (OperationModel and Process). More specifically, the ontology discusses:

- The sensor: To provide wide application of the ontology, Sensor has to include more than just the physical instrument but also the associated processing chain included in the measurement. It links together what it measures (the domain), the physical sensor (the grounding), and its functions and processing (the models).

- The concrete sensor: The Sensor Grounding models the concrete realization of a sensor. The grounding represents its physical implementation, including size, shape, materials, and location, as well as the concrete aspects of accessing data from the sensor, including the types and expected formats of input when calling functions, the format of output, and the details for accessing the sensor (e.g., radio, network, or physical access). The Operation Model describes how the sensor actually performs its measurement.

- The abstract sensor: The need to model single sensors, compound sensors, and larger processing chains and models suggests a continuum of abstractions for describing functions and processes. The Process concept models processes from abstract and atomic to compound, allowing for a single specification to be described at multiple levels of abstraction and to use multiple different types of abstractions in describing its processing.

These clusters cover a wider range of concepts than the other ontologies. It is able to describe most of the spectrum of sensor concepts, including composition and structure. The ontology can describe more sophisticated forms of structural and sequencing composition, with, for example, sequence, conditional, and repetition for process composition. A combination of OntoSensor (described in the next section) and this ontology represents the current level of expressive capability for semantic sensors. However, currently this ontology cannot describe the components of platforms. 


\subsection{Sensor Ontologies: From Shallow to Deep Models}

Russomanno et al. [13, 14] describe OntoSensor, an ontology intended as a general, knowledge base of sensors for query and inference. It is very general, and is comprised of definitions of concepts and properties adopted in part from SensorML [2] extensions to the Institute for Electrical and Electronics Engineers (IEEE) SUMO ontology [11], references to ISO $1911^{1}$, and constructs from the Web Ontology Language [1].

SensorML is a specification of a generic data model in Unified Modeling Language (UML) for capturing classes and associations that are common to all sensors. SensorML is part of an Open Geospatial Consortium (OGC) initiative to contribute to the development of a Sensor Web "through which applications and services will be able to access sensors of all types over the Web." ${ }^{2}$

OntoSensor extends the IEEE SUMO upper-level ontology by making some OntoSensor classes extensions of classes defined in SUMO. SensorML framework references some concepts that are defined in International Organization of Standardization (ISO) 19115, which defines schema required for geographic information and services.

Other relevant projects and articles that have not been further explored due to the fact that they appeared too far outside of the scope of manufacturing include:

- The Coastal Environmental Sensing Networks (CESN) project at the University of Massachusetts (Boston) is developing technology for sensor networks for coastal observing and is described in [15].

- The OOSTethys community is developing open-source resources to help install, integrate, and update standards-compliant Web services for oceanographic observing, with a particular emphasis on OGC standards and is described in [16].

\subsection{IEEE 1451.4}

IEEE 1451.5 [17] is a standard for adding plug and play capabilities to analog transducers. While not an ontology per se, it does provide a set of sensor specific data requirements that can be used to build the ontology.

The underlying mechanism for plug and play identification is the standardization of a Transducer Electronic Data Sheet (TEDS). A TEDS contains the critical information needed by an instrument or measurement system to identify, characterize, interface,

\footnotetext{
${ }^{1} \mathrm{http}: / /$ www.iso.org/iso/catalogue_detail.htm?csnumber=26020

${ }^{2}$ http://xml.coverpages.org/ni2008-01-31-a.html
} 
and properly use the signal from an analog sensor. The Basic TEDS uniquely identifies the transducer and includes the Manufacturer ID, model number, version letter, version number, and serial number of the device. Sensor specific templates are also included which include information such as minimum and maximum voltage output, resistance values, current, and temperature.

\section{Summary}

Each of the ontologies described in the previous section have elements useful in constructing a manufacturing perception sensor ontology, such as task assignment or provenance metadata. The two most general and comprehensive ontologies (as it pertains to the development of a manufacturing sensor ontology) appear to be the Neuhaus/Compton and OntoSensor efforts, and would be the most promising starts for building the manufacturing sensor ontology. The other efforts can give insights for the design of particular elements.

The next step in the NIST Sensor Ontology effort will be to further extend and refine the requirements by verifying the purposes of the ontology, reviewing the attributes in Section 2 for completeness, coverage, and redundancy, and putting them into appropriate hierarchies. Also, effort will be directed at choosing appropriate tools and languages for supporting the ontology, such as OWL and SensorML. After an initial design and choice of tools, the ontology will be implemented in the selected tool and validated on selected cases.

Once an initial version of the manufacturing perception sensor ontology is completed, it will be proposed as a standard within the IEEE Robotics and Automation Society (RAS) Ontologies for Robotics Automation (ORA) Working Group [3]. The goal of this working group is to develop a standard ontology and associated methodology for knowledge representation and reasoning in robotics and automation, together with the representation of concepts in an initial set of application domains. The standard provides a unified way of representing knowledge and provides a common set of terms and definitions, allowing for unambiguous knowledge transfer among any group of humans, robots, and other artificial systems. It is expected that the manufacturing perception sensor ontology will be developed and proposed in late 2013.

\section{Bibliography}

[1] F. Harmelen and D. McGuiness. (2004). OWL Web Ontology Language Overview, W3C web site: http://www.w3.org/TR/2004/REC-owl-features20040210/. 
[2] M. Botts and A. Robin, "Sensor Model Language (SensorML) implementation specification," 2007.

[3] C. Schlenoff, E. Prestes, R. Madhavan, P. Goncalves, H. Li, S. Balakirsky, T. Kramer, and E. Miguelanez, "An IEEE Standard Ontology for Robotics and Automation," presented at the International Conference on Intelligent Robots and Systems (IROS), Vilamoura, Algarve (Portugal), 2012.

[4] M. Compton, C. Henson, H. Neuhaus, L. Lefort, and A. Sheth, "A Survey of the Semantic Specification of Sensors," presented at the 2nd International Workshop on Semantic Sensor Networks, at 8th International Semantic Web Conference, 2009.

[5] A. Avancha, C. Patel, and A. Joshi, "Ontology-driven adaptive sensor networks," presented at the 1st Annual International Conference on Mobile and Ubiquitous Systems, Networking and Services, 2004.

[6] C. Matheus, D. Tribble, M. Kokar, M. Ceruti, and S. McGirr, "Towards a formal pedigree ontology for level-one sensor fusion," presented at the 10th International Command \& Control Research and Technology Symposium, 2005.

[7] M. Gomez, A. Preece, M. Plohnson, G. d. Mel, W. Vasconcelos, C. Gibson, A. BarNov, K. Borowiecki, T. L. Porta, and D. Pizzocaro, "An ontology-centric approach to sensor- mission assignment," presented at the 16th International Conference on Knowledge Engineering and Knowledge Management, 2008.

[8] A. Preece, M. Gomez, G. d. Mel, W. Vasconcelos, S. Sleeman, S. Colley, G. Pearson, T. Pham, and T. L. Porta, "Matching sensors to missions using a knowledge-based approach," presented at the SPIE Defense Transformation and Net-Centric Systems, 2008.

[9] M. Eid, R. Liscano, and A. E. Saddik, "A novel ontology for sensor networks data," presented at the IEEE International Conference on Computational Intelligence for Measurement Systems and Applications, 2006.

[10] M. Eid, R. Liscano, and A. E. Saddik, "A universal ontology for sensor networks data," presented at the IEEE International Conference on Computational Intelligence for Measurement Systems and Applications, 2007.

[11] A. Pease, I. Miles, and J. Li, "The Suggested Upper Merged Ontology: A Large Ontology for Semantic Web and its Applications," in Working Notes of the AAAI-2002 Workshop on Ontologies and the Semantic Web, Edmonton, Canada, 2002.

[12] H. Neuhaus and M. Compton, "The semantic sensor network ontology: A generic language to describe sensor assets," presented at the AGILE Workshop: Challenges in Geospatial Data Harmonization, 2009.

[13] D. Russomanno, C. Korthari, and O. Thomas, "Building a sensor ontology: "A practical approach leveraging ISO and OGC models," presented at the 2005 International Conference on Artificial Intelligence, 2005.

[14] D. Russomanno, C. Kothari, and O. Thomas, "Sensor ontologies: from shallow to deep models," presented at the Thirty-Seventh Southeastern Symposium on System Theory, 2005. 
[15] T. D. C. Little, J. Knorad, and P. Ishwar, "A Wireless Video Sensor Network for Autonomous Coastal Sensing," presented at the Conference on Coastal Environmental Sensing Networks (CESN), 2007.

[16] E. Bridger, L. E. Bermudez, M. Maskey, C. Rueda, B. L. Babin, and R. Blair, "OOSTethys - Open Source Software for the Global Earth Observing Systems of Systems," presented at the American Geophysical Union, Fall Meeting 2009.

[17] IEEE, "IEEE standard for a smart transducer interface for sensors and actuators wireless communication protocols and transducer electronic data sheet (TEDs) formats - IEEE Std 1451.5," ed, 2007, pp. C1 - 236. 